\title{
Is Kinesio taping better than placebo taping for improving performance during unilateral vertical jump and hop tests? Protocol study for a randomized, placebo-controlled, double-blind, clinical trial
}

\author{
Igor Phillip dos Santos Glória',2, Carolina Marciela Herpich', Felipe Serenza², Fabiano Politti', \\ Tabajara de Oliveira Gonzalez' ${ }^{1}$ Cid André Fidelis de Paula Gomes', Daniela Aparecida Biasotto-Gonzalez
}

\begin{abstract}
Introduction: Kinesio taping consists of the attachment of a thin elastic tape over specific muscles, the thickness of which is similar to that of the epidermis. This tape can be stretched to $140 \%$ of its original length, which demonstrates greater elasticity in comparison to conventional bandages. According to the creator of this method, Kinesio taping leads to better muscle activation or inhibition, reduces pain, swelling and muscle spasms and prevents muscle injury. Objective: The aim of the proposed study is to compare and analyze the immediate effect of Kinesio taping and placebo taping on performance during unilateral vertical jump and two hop tests by young professional soccer players. Methods: A randomized, placebo-controlled, double-blind, clinical trial will be conducted involving 100 athletes. The volunteers will be submitted to an evaluation of the dominant lower limb using the Single Leg Hop Test and Single Leg Triple Hop Test as well as the evaluation of a unilateral vertical jump on a pressure platform. The athletes will then be randomly allocated to two groups. Group A will receive Kinesio taping of the triceps surae muscle as described by Kase (2003, 2013) and Group B will receive a placebo taping of the same muscle. After 30 minutes, the volunteers will be submitted to the same evaluation tests. Analysis: Intra-group and inter-group analyses of the results will be performed. Statistical tests will be conducted considering a $5 \%$ significance level. This study is registered with Clinical Trials under protocol number: NCT02560961
\end{abstract}

Key words: Kinesio Taping; Soccer; Hop Test; Vertical Jump

Trial registry: Clinical Trials

Registration number: NCT02560961

\section{INTRODUCTION}

Kinesio taping was invented by Kenzo Kase in 1996. ${ }^{(1)}$ The technique consists of the attachment of a thin elastic tape over a specific muscle, the thickness of which is similar to that of the epidermis. This tape can be stretched to $140 \%$ of its original length, which demonstrates greater elasticity in comparison to conventional bandages. According to the creator, Kinesio taping reduces pain, swelling and muscle spasms and prevents injury. ${ }^{(1)}$

Kinesio Taping is currently used to prevent muscle injuries in athletes. ${ }^{(2)}$ This technique is reported to enhance muscle strength and increase electromyographic activity, with such effects lasting two days after the removal of the tape. Other benefits include a reduction in pain, improved gait pattern and improved function in athletes as well as individuals with osteoarthritis and femoropatellar pain syndrome. ${ }^{(3-5)}$
In recent years, clinical trials and case studies have employed Kinesio taping for the increase in muscle strength, ${ }^{(6)}$ reduction in pain. ${ }^{(7,8)}$ However, recent systematic reviews on the effects of Kinesio taping have found no evidence of such benefits. Indeed, Parreira et al..$^{(9)}$, concluded that there is no scientific evidence to support the use of this method in clinical practice. Csapo \& Alegre ${ }^{(10)}$, report that, although Kinesio taping may have some benefits, it is not capable of improving muscle performance in healthy adults.

Physiological explanations for the benefits of Kinesio taping are scarce. This technique is thought to facilitate and stimulate muscle function. For such, the application of the tape should begin at the origin of the muscle and end at the point of insertion. The elastic properties of the tape are thought to stimulate the muscle in the direction of the contraction, 
thereby enhancing movement. Hypothetically, Kinesio taping may stimulate mechanoreceptors in the skin and increase the recruitment of motor units. Another hypothesis is that Kinesio taping may stimulate the fascia in the muscle and cause greater muscle tension, thereby facilitating contraction. ${ }^{(11)}$

Physiological explanations are based on empirical evidence. Thus, studies with adequate methodological quality are needed to establish the real effects of Kinesio taping, especially with regard to muscle activation in athletes, who constitute the population that most employs this technique, even during high-performance athletic competitions. ${ }^{(12)}$

The aim of the proposed study is to analyze the effect of Kinesio taping on the activation of the triceps surae muscle and performance during unilateral vertical jump and two hop tests by young professional soccer players.

\section{Hypothesis}

The hypothesis of this study is that Kinesio taping enhances the performance on unilateral vertical jump and hop tests.

Primary outcome: performance on unilateral vertical jump Secondary outcome: performance on hop tests

\section{METHODS}

\section{Overview of research design}

A randomized, placebo-controlled, double-blind, clinical trial will be conducted to determine the effect of Kinesio taping on the triceps surae muscle of the dominant lower limb on performance during a unilateral vertical jump as well as both the Single Leg Hop Test and Single Leg Triple Hop Test.

\section{Approval and registry}

The project received approval from the Human Research Ethics Committee of University Nove de Julho (Sao Paulo, Brazil) under process number 26316414.4.0000.5511 and will be conducted at the Human Movement Analysis Laboratory of the same university. This study has been registered with ClinicalTrials. gov (NCT02560961).

\section{Participants}

The study will be conducted with all professional soccer players of the categories, under 15, 17 and 20 of RedBull soccer Brazil, totaling 100 athletes. Those who agree to participate and meet the eligibility criteria will be selected for the study. Authorization from parents/guardians will be requested for volunteers less than 18 years of age. This study will be conducted in compliance with the norms regulating studies involving human subjects stipulated in Resolution $n^{\circ} 196 / 97$ of the Brazilian National Board of Health.

\section{Eligibility criteria}

\section{Inclusion criteria}

Practitioners of soccer aged 15 to 20 years with a body mass index (BMI) within the ideal range (20 to $\left.25 \mathrm{Kg} / \mathrm{m}^{2}\right)$.

\section{Exclusion criteria}

History of musculoskeletal injury in the lower limbs in the previous six months and history of surgery on the lower limbs or back in the previous six months.

\section{Evaluation procedures}

The Consolidated Standards of Reporting Trials - CONSORT ${ }^{(13)}$ will be used for the proposed randomized clinical trial.

Personal data will be collected using a chart designed by the authors. This chart will contain the following information: name, date of birth, dominant limb, weight and height. Weight and height will be determined using a digital scale (Worker ${ }^{\circledR}$ ) and stadiometer (Sanny ${ }^{\circledR}$ ), respectively. The dominant limb will be recorded based on the volunteer's answer to a question regarding which leg he would use for a penalty kick. The BMI will be calculated based on weight and height. Individuals with a BMI greater than $25 \mathrm{Kg} / \mathrm{m}^{2}$ or less than $20 \mathrm{Kg} / \mathrm{m}^{2}$ will be excluded to standardize the sample.

All volunteers will then undergo the following evaluations:

\section{- Unilateral vertical jump:}

The unilateral vertical jump will be used to measure performance, as this movement requires triceps surae strength $^{(14)}$. The vertical jump will be evaluated using a pressure plate (BIOMEC 400 v1.1 ${ }^{\circledR}$ EMG Systems do Brasil) connected to computer (Inter Core 2 Dual $^{\circledR}$ ) with $2 \mathrm{GHz}$ and 250 Giga bytes. This system quantifies the distribution of vertical ground reaction force in a static or dynamic position with a sampling frequency of $40 \mathrm{~Hz}$ using four force sensors (two anterior and two posterior) capable of supporting $150 \mathrm{Kg}$. Each sensor stores analog data, which are amplified and converted to digital data interpreted using the Biomec $400^{\circledR}$ software program (EMG Systems do Brasil). This system records the distribution of force among the four points and changes in body sway in the anteroposterior ( $\mathrm{Y}$ axis) and mediolateral ( $\mathrm{X}$ axis) directions. The force plate will be positioned on an even surface. Levelness will be determined by two levels attached to the extremities of the force plate. The volunteer will be instructed to stand on the dominant leg with hands on the hips and jump as high as possible, landing on the same leg (Figure 1). Initially, three jumps will be performed to familiarize the volunteer with the procedure, with a one-minute rest interval between jumps. After a two-minute rest interval, three additional jumps will be performed with a one-minute rest interval between jumps, which will be considered for evaluation. Jump time will be determined using the following formula:

$$
h=0.5(t / 2)^{2} g
$$

in which $\mathrm{h}=$ jump height in meters, $\mathrm{t}=$ jump time in seconds and $\mathrm{g}=$ acceleration of gravity $\left(9.81 \mathrm{~m} / \mathrm{s}^{2}\right)$. 
- Single Leg Hop Test

Test commonly used to measure functional performance and progression during lower limb rehabilitation programs ${ }^{(15-17)}$, with the distance achieved during a single, one-foot hop recorded (Figure 2$)^{(16)}$. For such, the athlete will stand only on the dominant leg behind a line marked on the floor and will hop as far as possible without using the contralateral leg and without losing balance. The distance will be measured with a metric tape from the line marked on the floor to the most proximal point of the heel landing on the floor at the end of the hop. ${ }^{(18)}$ The volunteers will be instructed to keep their hands on their hips throughout the test to reduce the influence of the upper limbs on the performance of the hop test. Initially, three hops will be performed to familiarize the volunteer with the procedure, with a one-minute rest interval between hops. After a two-minute rest interval, three additional hops will be performed with a one-minute rest interval between hops, which will be considered for evaluation.

\section{- Single Leg Triple Hop Test}

The volunteer will also be positioned on the dominant leg behind the line marked on the floor and will perform three consecutive hops as far as possible without using the contralateral leg and without losing balance. The final distance will be measured with a metric tape from the line marked on the floor to the most proximal point of the heel landing on the floor at the end of the third hop (Figure 2$)^{(18)}$. The volunteers will be instructed to keep their hands on their hips throughout the test to reduce the influence of the upper limbs on the performance of the hop test.

On both hop tests, landing must be made in a stable fashion without touching the contralateral leg or upper limbs to the floor for a hop to be considered valid.

\section{Frequency of evaluations}

Evaluations will be conducted in the following manner:

Phase I:

- Five minutes of warm up on a stationary bike;

- Unilateral vertical jump on a force plate (3 jumps);

- Single Leg Hop Test and Single Leg Triple Hop Test (three repetitions of each test);

- Randomization and allocation of individuals to respective groups.

Phase II:

- 30 minutes after intervention:

- Five minutes of warm up on a stationary bike;

- Unilateral vertical jump on a force plate (3 jumps);
- Single Leg Hop Test and Single Leg Triple Hop Test (three repetitions of each test).

\section{Blinding procedures}

Following the initial evaluations (Figure 3), the athletes will be randomly allocated to two experimental groups stratified by age. Randomization will be performed with sealed opaque envelopes with cards stipulating to which group each participant will belong. The participants will remain blinded to the allocation. For such, the tape will be applied with the volunteer in the prone position and his view blocked during the procedure. A sock will then be placed over the lower portion of the leg, covering the entire region in which the tape was applied. One researcher will be in charge of the randomization process and application of the tape to the dominant leg of the volunteers and will not participate in the evaluations. Another researcher will perform the evaluations and will be blinded to the allocation of the volunteers to the different groups. The statistician will also be blinded to the allocation of the participants until the completion of the statistical analysis.

\section{Intervention procedures}

\section{Kinesio Taping protocol - Group A}

Kinesio Taping (Kinesio Tex Gold ${ }^{\circledR}$; color: black) will be applied by an experienced, duly trained researcher. The technique described by Kase et al. ${ }^{(1)}$ for activation of the triceps surae on the dominant leg will be employed, with the volunteer in the prone position, maintaining the dominant leg off the cot with hip extension, knee extension and ankle dorsiflexion to maintain the triceps surae in a stretched position. The tape will be applied in a Y shape beginning with the origin of the muscle (femur condyles) without tension (Anchor I), passing over the belly of the muscle (therapeutic region) with 15 to $20 \%$ tension and ending near the posterior surface of the heel (Anchor II) without tension (Figure 4).

\section{Placebo Taping - Group B}

Standard white bandaging tape will be applied by the same researcher who placed the tape in Group A. The technique described by Kase et al. (1) for activation of the triceps surae on the dominant leg will be employed, with the volunteer in the prone position, maintaining the dominant leg off the cot with hip extension, knee extension and ankle dorsiflexion to maintain the triceps surae in a stretched position. The tape will be applied in a $Y$ shape beginning with the origin of the muscle (femur condyles) and ending near the posterior surface of the heel (Figure 4).

\section{Statistical analysis}

The data will be organized in tables and graphs using mean and standard deviation values for each variable. The data will be submitted to parametric and/or nonparametric statistical 
tests, depending on normal or non-normal distribution. A $p$-value $p \leq 0.05$ will be indicative of statistical significance.

\section{DISCUSSION}

This study will be conducted to investigate the effect of Kinesio taping on the activation of the triceps surae muscle in soccer players. This muscle is highly demanded for activities that involved jumping. Unilateral vertical jumping on a force plate and two hop tests will be used to evaluate the performance of this muscle.

Although Kinesio taping is often used for the treatment of musculoskeletal problems and to enhance muscle performance in sports, few studies have demonstrated such physiological effects.

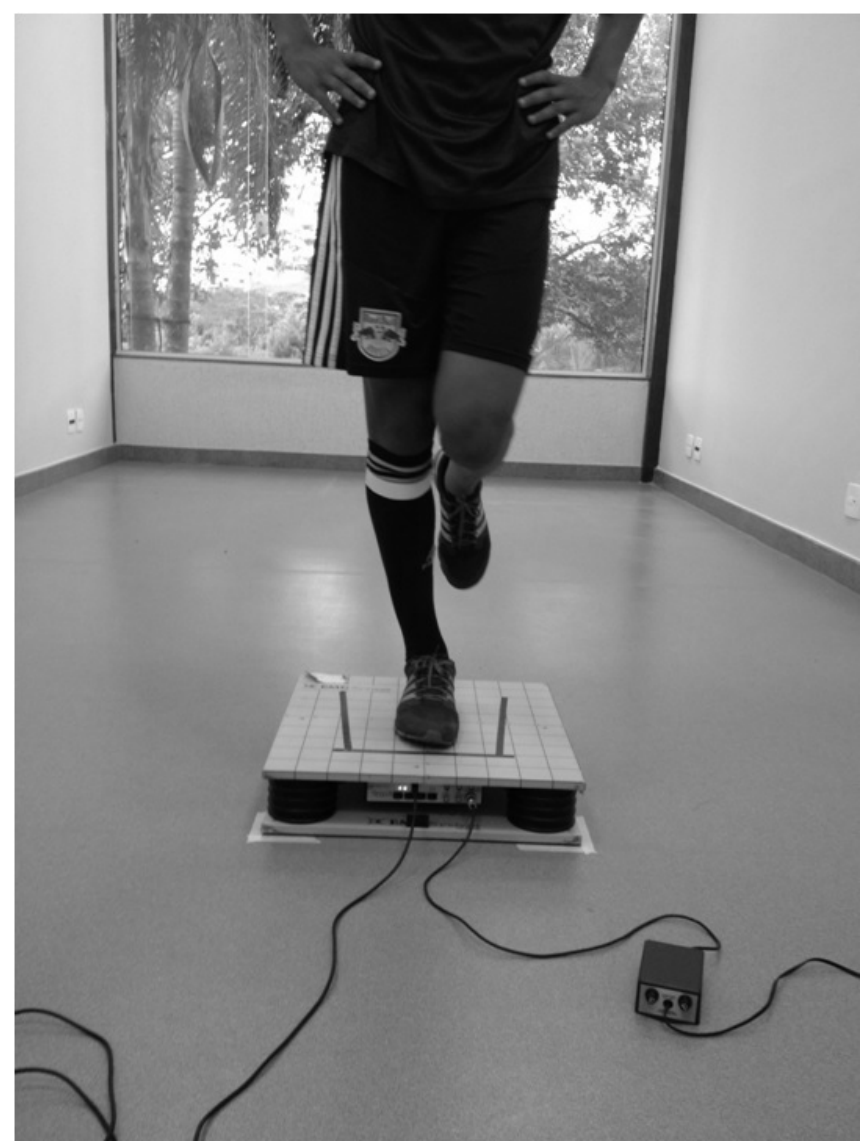

Figure 1. Positioning of volunteer on force plate for unilateral vertical jump.

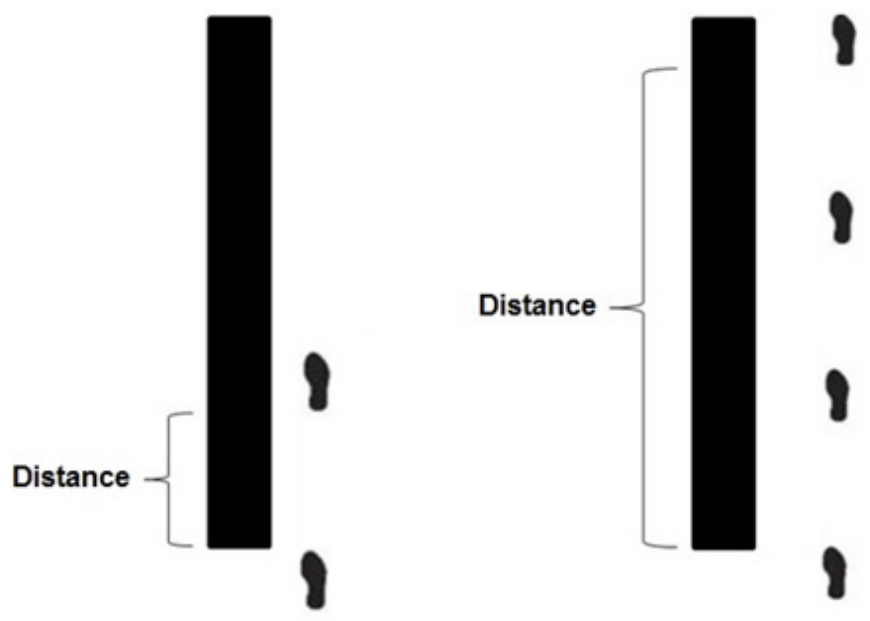

Figure 2. Representation of Single Leg Hop Test (left side) and Single Leg Triple Hop Test (right side) 


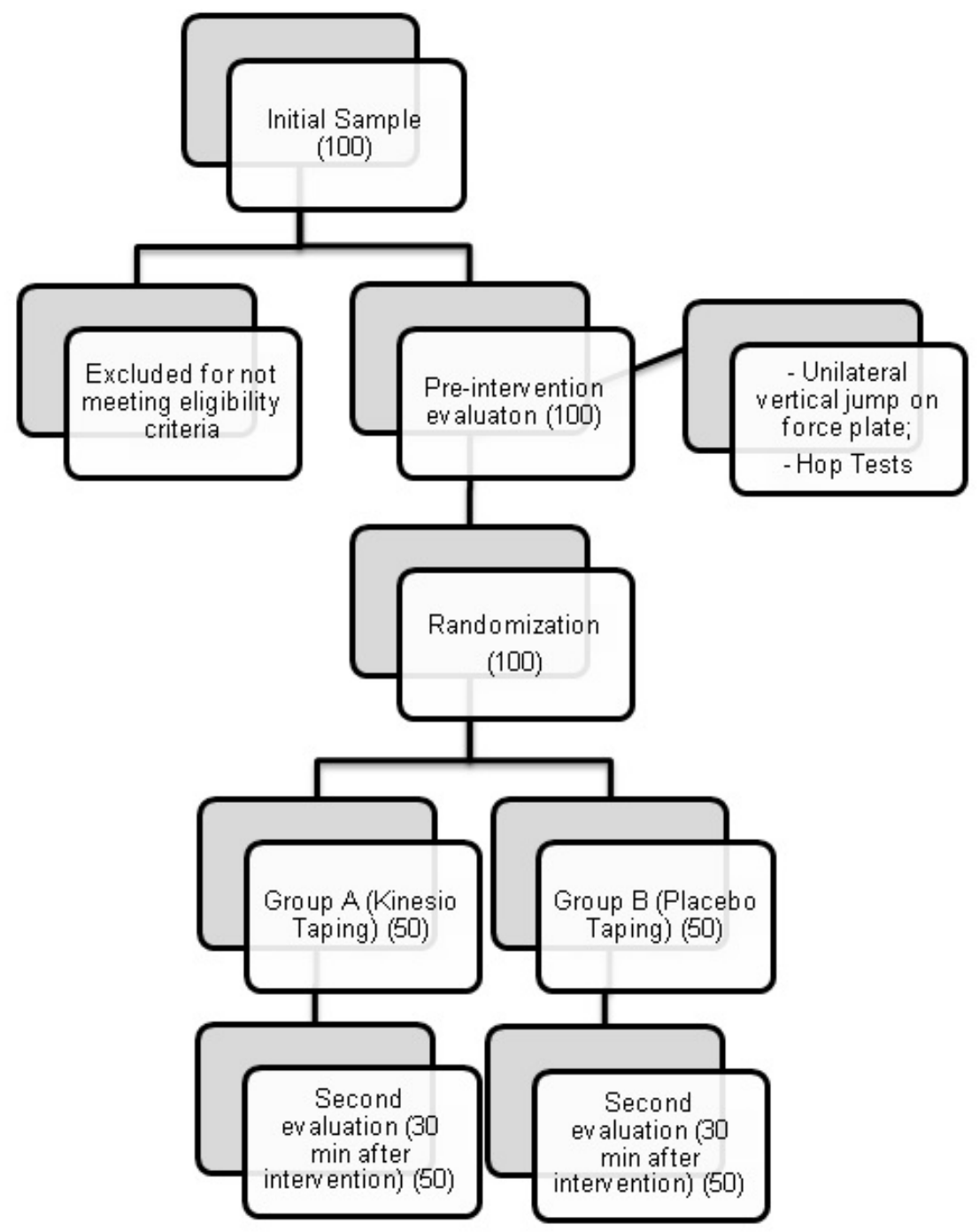

Figure 3. Flowchart of study (CONSORT, 2010)

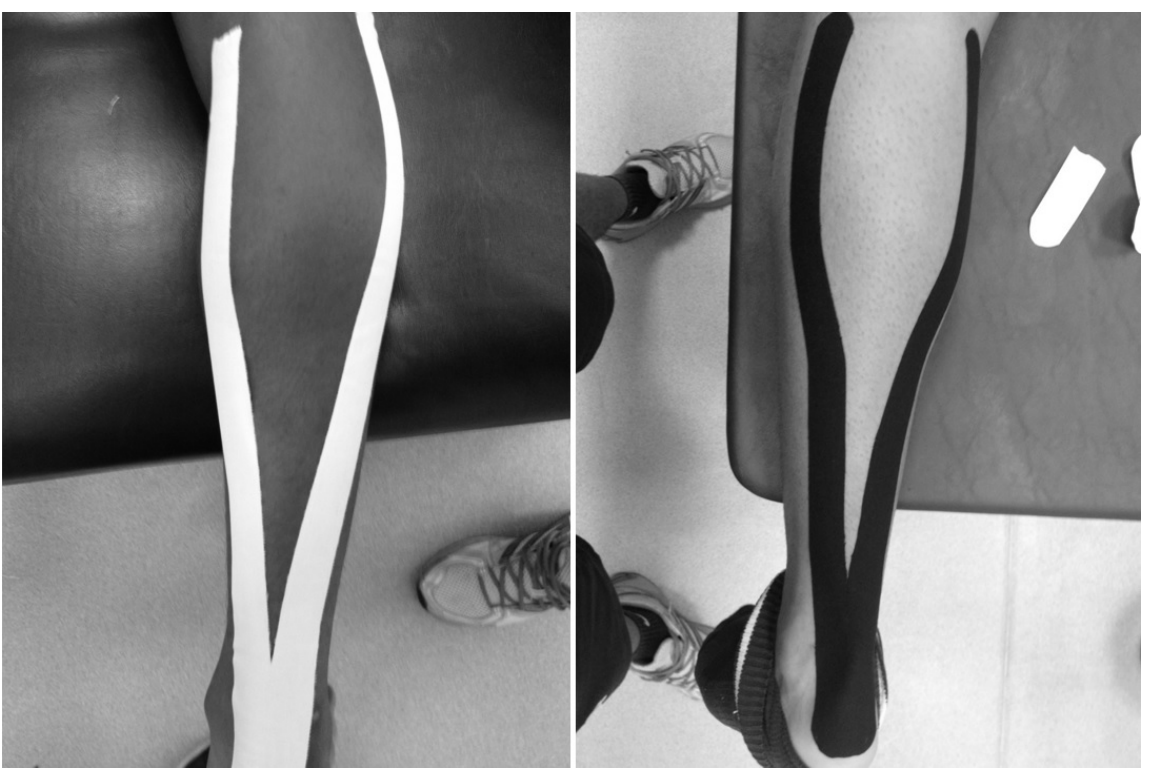

Figure 4. Application of Kinesio taping (Group A) and placebo taping (Group B) 


\section{AUTHORS' CONTRIBUTIONS:}

IPSG: Design, development of research and writing of text; $\mathrm{CMH}$ : Data collection; FS: Data collection; FP: Statistical analysis; TOG: Planning of the methodological design; CAFPG: Planning of the methodological design; DABG: Supervision and critical review

\section{COMPETING INTERESTS:}

The author(s) declare that they have no competing interests.

\section{AUTHOR DETAILS}

${ }^{2}$ Redbull Brazil Soccer, Campinas (SP), Brasil.

\section{REFERENCES}

1. Kase K, Wallis J, Kase T. Clinical Therapeutic Applications of Kinesio Taping Method. Tokyo, Ken Ikai Co Ltda., 2003.

2. Cools AM, Witvrouw EE, Danneels LA, Cambier DC. Does taping influence electromyographic muscle activity in the scapular rotators in healthy shoulders? Manual Ther. 7: 154-62. 2002.

3. Hinman RS, Bennell KL, Crossley KM, MacConnell J. Immediate effects of adhesive tape on pain and disability in individuals with knee osteoarthritis. Rheumatology. 42: 865-9. 2003.

4. Ernst GP, Kawaguchi J, Saliba E. Effect of patellar taping on knee kinetics of patients with patellofemoral pain syndrome. J Orthop Sports Phys Ther; 29: 661-7, 1999.

5. Słupik A, Dwornik M, Białoszewski D, Zych. Effect of Kinesio Taping on bioelectrical activity of vastus medialis muscle. Preliminary Report Ortop Traumatol Rehabil; 9(6):644-651. 2007.

6. Fratocchi G., Di Mattia F, Rossi R, Mangone M, Santilli V, Paoloni M. Influence of Kinesio taping applied over biceps brachii on isokinetic elbow peak torque. A placebo controlled study in a population of young healthy subjects. Journal of Science and Medicine in Sport 16: 245-49, 2013.
7. Lee J, Yoo W. Application of posterior pelvic tilt taping for the treatment of chronic low back pain with sacroiliac joint dysfunction and increased sacral horizontal angle. Physical Therapy in Sport 13: 279 - 28, 2012.

8. Lee J, Yoo W. Treatment of chronic Achilles tendon pain by Kinesio taping in an amateur badminton player. Physical Therapy in Sport 13: 115 - 119, 2012.

9. Parreira P C E, Costa L C M, Junior L C H, Lopes A D, Costa L O P. Current evidence does not support the use of Kinesio taping inclinical practice: a systematic review. Journal of Physiotherapy. 60: 31-39, 2014.

10. Csapo R, Alegre L M. Effects of Kinesio ${ }^{\circledR}$ taping on skeletal muscle strength - A meta-analysis of current evidence. Journal of Science and Medicine in Sport. S/P online, 2014.

11. Nunes GS, de Noronha M, Cunha HS, Ruschel C, Borges NG Jr. Effect of kinesio taping on jumping and balance in athletes: a crossover randomized controlled trial. J Strength Cond Res. 2013 Nov;27(11):3183-9.

12. Kalron A, Bar-Sela S. A systematic review of the effectiveness of Kinesio Taping fact or fashion? Eur J Phys Rehabil Med. 2013 Oct;49(5):699-709. Epub 2013 Apr 5.

13. Schulz, K. F. Altman,D. G., Mohe, D. CONSORT 2010 Statement: updated guidelines for reporting parallel group randomised trials CONSORT Statement, 2010

14. Huang CY, Hsie TH, Lu SC, Su FC. Effect of the Kinesio tape to muscle activity and vertical jump performance in healthy inac- tive people. Biomed Eng online $2011 ; 10: 70$.

15. Fitzgerald, G. K., Lephart S.M., Hwang, J.H., Wainner, M.R.S. Hop Tests as Predictors of Dynamic Knee Stability. Journal of Orthopaedic \& Sports Physical Therapy. 31: 588-597, 2001.

16. Kramer J.N.D., Power M., Webster-Bogaert S., Test Retest of the One Leg Hope Test Following ACL Reconstruction. Clinical Journal of Sports Medicine. 2: 240-43, 1992.

17. Rudolph K.S., Axe M.J., Snyder-Mackler L., Dynamic Stability after ACL injury: whon can hop? Knee Surg Sports Trau Arthrosc, 8: 262-269, 2000.

18. Logerstedt, et al. Single-Legged Hop Tests as Predictors of Self-Reported Knee Function After Anterior Cruciate Ligament Reconstruction. The American Journal of Sports Medicine,40(10):2012. 